\section{Cahiers de Narratologie}

Analyse et théorie narratives

17 | 2009

Stéréotype et narration littéraire

\title{
Le stéréotype du bandit catalan dans la littérature espagnole du Siècle d'Or
}

\section{Mathias Ledroit}

\section{(2) OpenEdition}

\section{Journals}

Édition électronique

URL : http://journals.openedition.org/narratologie/1297

DOI : 10.4000/narratologie.1297

ISSN : 1765-307X

Éditeur

LIRCES

\section{Référence électronique}

Mathias Ledroit, «Le stéréotype du bandit catalan dans la littérature espagnole du Siècle d'Or », Cahiers de Narratologie [En ligne], 17 | 2009, mis en ligne le 22 décembre 2009, consulté le 14 novembre 2019. URL : http://journals.openedition.org/narratologie/1297 ; DOI : 10.4000/narratologie. 1297

Ce document a été généré automatiquement le 14 novembre 2019.

\section{c) (†) $९$}

Cahiers de Narratologie - Analyse et théorie narratives est mis à disposition selon les termes de la licence Creative Commons Attribution - Pas d'Utilisation Commerciale - Pas de Modification 4.0 International. 


\title{
Le stéréotype du bandit catalan dans la littérature espagnole du Siècle d'Or
}

\author{
Mathias Ledroit
}

1 Personnage terrifiant et mythique ${ }^{1}$, le bandit a envahi le monde artistique de l'Antiquité à nos jours. Les écrivains espagnols du Siècle d'Or lui ont consacré de nombreuses pages, voire des œuvres entières à une époque où le banditisme est à son faîte dans certaines provinces de la monarchie espagnole et notamment en Catalogne qui a donné à la littérature classique ses plus célèbres bandits : Roque Guinart, dans le Quichotte; Antonio Roca, personnage éponyme d'une comedia de Lope de Vega ; et Pedro Armengol dans El Bandolero de Tirso de Molina².

Tous les bandits ne sont certes pas catalans, mais l'association entre la Principauté et le banditisme revient fréquemment dans les textes, comme l'illustre l'œuvre de Miguel de Cervantès où ce personnage emblématique surgit immanquablement dans les intrigues qui se déroulent en Catalogne. Ainsi dans Les deux jeunes filles, don Rafaël et Téodisia croisent-ils un groupe de voyageurs ayant été attaqués par des bandits à « deux lieues d'un bourg qui est à neuf lieues de Barcelone, et qu'on appelle Igualada ${ }^{3}$ ». De même, dans la seconde partie de ses aventures, l'Ingénieux Hidalgo ne déduit-il pas qu'il se trouve près de Barcelone en comprenant que les jambes qui pendent des arbres et qui ont effrayé Sancho sont celles de "quelques bannis et bandoliers, que l'on a pendus à ces arbres, car c'est ici que la justice a accoutumé à les pendre quand elle les peut attraper ${ }^{4}$ ». À propos de cette réplique, Martín de Riquer a écrit que les lecteurs qui découvraient le second tome du Quichotte en 1615 avaient dû parvenir à la même conclusion tant banditisme et Catalogne ne faisait qu'un dans l'esprit des Castillans du XVII ${ }^{e}$ siècle ${ }^{5}$.

3 Cette analyse concorde avec la définition qu'a donnée Odile Gannier du stéréotype, à savoir qu'il est une association stable d'éléments formant une unité ressentie comme nécessaire à un moment précis de l'action ${ }^{6}$. Le rapprochement entre le banditisme et la Catalogne ne se décline pas uniquement sous la forme exposée précédemment. On 
trouve également des bandits catalans à la tête d'escouades castillanes, comme dans $E l$ Peregrino en su patria de Lope de Vega ${ }^{7}$ où il est fait allusion à un groupe de bandoleros qui «militaient sous la protection et l'étendard d'un chevalier catalan », car c'est, selon Tirso de Molina dans Los Cigarrales de Toledo, « une coutume dans cette principauté8 ${ }^{\text {». }}$

S'il existe un stéréotype littéraire comme semblent l'indiquer ces quelques remarques préliminaires, le personnage du bandit n'appartient pas seulement à l'univers artistique et nombreux sont les textes historiques, géographiques ou juridiques du $\mathrm{XVII}^{\mathrm{e}}$ siècle qui renvoient l'image d'une Catalogne envahie de bandits9. Ces mêmes textes se sont ainsi attachés à décrire le phénomène du banditisme non comme le résultat d'une mauvaise conjoncture, mais comme celui du tempérament des habitants de cette province plus enclins, selon eux, à la pratique du banditisme. Peut-on dès lors établir quelque lien entre le poncif littéraire et le stéréotype social ? Le second aurait-il fait émerger le premier?

5 Il s'agira ici d'analyser le poncif du bandit catalan dans certaines des œuvres de Cervantès, de Lope de Vega et de Tirso de Molina en mettant en lumière les ressemblances qui existent entre le personnage littéraire et le stéréotype social pour pouvoir ensuite s'interroger sur l'interprétation qu'il faut faire de cet archétype catalan.

Analyse du poncif

Bandolero: celui qui se réfugie dans la montagne en emmenant avec lui des hommes de son clan. Ils abandonnent leur maison et leurs terres pour se venger de leurs ennemis. Parce qu'ils sont nobles, ils ne tuent aucune des personnes qu'ils rencontrent, bien que, pour survivre, ils les dépouillent d'une partie de leurs biens. D'autres bandouliers sont en revanche des bandits de grands chemins qui ne se contentent pas de dépouiller les voyageurs, mais qui les maltraitent et les tuent ${ }^{10}$.

Cette définition du Tesoro de la lengua castellana de Sebastián de Covarrubias montre que la langue classique distingue rigoureusement le bandolero du voleur qu'elle désigne plus volontiers par les termes de "ladrón» ou "salteador de camino». Cette distinction lexicale montre qu'il existe dans le monde du banditisme deux figures qui coexistent tout en présentant des différences notables. Tandis que le voleur ne vit que pour attaquer les convois ou les personnes seules qu'il peut arrêter sur les chemins, le bandoulier ne conçoit le vol que comme un moyen de survie. Les scènes de braquage sont toujours l'occasion de dévoiler le stéréotype de bandit au grand cœur. Sans jamais avoir recours à la violence, il ne dérobe que le minimum nécessaire pour contenter son bataillon en répartissant équitablement le butin, ce qui lui vaut les louanges de ses victimes et les reproches de ses hommes de suite qui le considèrent "plutôt fait pour être frade que brigand ${ }^{11}$ ». Leur intention, en effet, "n'est pas selon Roque Guinart de charger les soldats, ni les femmes, principalement celles qui sont de qualité ${ }^{12}$ ", mais, pour reprendre les termes d'Antonio Roca, de «nourrir avec cent écus/cette escouade de soldats ${ }^{13}$ ». De plus, le bandit n'hésite pas à redistribuer ce qui reste à ses victimes, comme Roque Guinart qui, ému par la tristesse des pèlerins, ordonne que

De ces écus il en revient deux à chacun de vous, et il en reste vingt ; qu'on en donne dix à ces pèlerins, et les autres dix à cet écuyer, afin qu'il puisse dire du bien de cette aventure ${ }^{14}$.

7 Le bon souvenir qu'il entend laisser aux pèlerins est destiné à accroître sa renommée et sa popularité. La distinction est plus évidente encore dans l'intermède de La Cueva de Salamanca de Cervantès où l'étudiant, qui a eu affaire aux bandits en traversant la Catalogne, s'écrie en arrivant chez lui qu'il a été laissé sans le sou par les écuyers de 
Roque Guinart " parce qu'il était absent; eût-il été présent, il n'eût consenti qu'on me fît cette offense, car il est très courtois et modéré et, de surcroît, généreux ${ }^{15}$ ».

8 La différence entre ces deux personnages se creuse davantage dans le sentiment d'antipathie envers le voleur que le texte littéraire traduit et qui renforce l'image positive du bandit qui lui-même se méfie de ses écuyers. Dans le chapitre LXI de la seconde partie du Quichotte, on apprend effectivement que Roque Guinart « passait la nuit séparé des siens ", car « il craignait [qu'ils] n'entreprissent sur sa vie ou qu'ils le livrassent entre les mains de la justice ${ }^{16}$ ", ce qui arrive à Antonio Roca dans la troisième journée de la comedia où il est dénoncé par un des ses hommes, Leonido, qu'il avait réprimandé sévèrement :

[...] en te livrant au vice-roi

je me vengerai de cette offense ${ }^{17}$.

9 Cependant, le bandoulier ne pourrait exister sans ses hommes de suite, car il appartient à un clan dont il a pris le commandement par la force. Invariablement, le bandit est un homme au physique imposant, même si cette image ne reflète pas nécessairement la réalité historique. Lluís Maria Soler i Terol a retrouvé des témoignages de personnes ayant vu ou aperçu Perot Rocaguinarda qu'ils décrivaient comme un homme grand et dégingandé18, alors que dans le Quichotte, il apparaît comme quelqu'un de "robuste, de taille plus que moyenne ». Cette entorse à la réalité montre bien que l'aspect physique du bandit est une caractéristique essentielle du personnage littéraire que l'on retrouve sans surprise chez Antonio Roca, dont la mère dit qu'il ressemblait à « un monstre » à sa naissance ${ }^{19}$ et chez Pedro Armengol qui est élégant et robuste ${ }^{20}$. Cette supériorité physique permet au bandit d'imposer son autorité au milieu des personnages peu recommandables avec qui il doit vivre. Dans Antonio Roca, le personnage éponyme prend la tête d'une faction suite au combat qu'il livre contre quatre chefs qu'il croise pendant sa fuite. Ces derniers, parce qu'ils ont été tenus en respect par un seul homme, l'implorent de prendre le commandement :

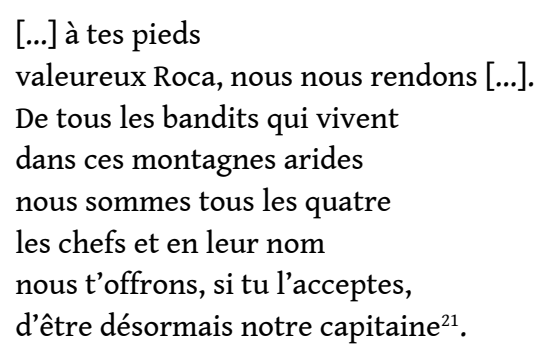

10 Dans le Quichotte, Roque Guinart lui aussi fait usage de la force pour faire respecter son autorité. Lorsque l'un de ses hommes ose critiquer sa générosité, il lui fend la tête en deux puis s'écrie :

Voilà comment je châtie les babillards et les téméraires ${ }^{22}$.

11 On pourrait juger ce recours systématique à la violence comme paradoxal, voire incompatible avec l'image d'un bandit généreux; mais le bandoulier vit dans un univers où il n'est d'autre loi que celle du plus fort. Comme le suggère l'étymologie de son nom, le bandit est un hors-la-loi : il est au ban ${ }^{23}$ de la société, condamné à vivre dans un monde où la justice civile ne peut l'atteindre. Contrairement au voleur qui a quitté la ville, et donc la société, pour des raisons économiques, le bandit, lui, est en fuite à cause d'un meurtre. Généralement, les motifs du crime sont exposés et constituent l'élément déclencheur de l'intrigue. Dans Antonio Roca, le héros, un religieux qu'a priori rien ne destinait au banditisme, avoue que «ce n'est pas tant 
l'appât du gain qui a provoqué mon impulsion que le triste désir de venger une mort et une offense $\mathrm{e}^{24}$ ». La mort à laquelle il fait allusion est celle de son père, assassiné par le baron Alverino alors qu'il tentait de secourir son épouse enlevée et déshonorée par le baron. Dans El bandolero, la situation est différente : Pedro Armengol, un jour qu'il se trouvait à Barcelone, aperçoit un Gascon en train de jeter des pierres sur la statue en cristal du roi Jaime II, ce qu'il assimile à un crime de lèse-majesté. N'écoutant que ce que son honneur lui dicte, il tue le criminel et s'enfuit par les rues obscures de la capitale. Dans le Quichotte, en revanche, on ne sait rien des motivations de Roque Guinart, sinon qu'il doit sa situation à « je ne sais quels désirs de [se] venger qui ont la puissance de troubler les cœurs les plus calmes ${ }^{25}$ ».

Le désir de vengeance semble complètement dépasser les personnages. Dans $E l$ bandolero, Tirso de Molina met l'accent sur la fatalité, puisqu'à la naissance de Pedro Armengol, deux astrologues confirment à son père qu'il est appelé à devenir chef de faction. Dans l'espoir de contredire les astres, Alberto Armengol confie vainement son enfant à une famille de paysans dont il prendra le nom pour s'appeler Pedro Guillén. Antonio Roca, quant à lui, lutte contre son désir de vengeance, mais ses réticences finissent par céder face à la question du point d'honneur dont on connaît le rôle primordial dans l'Espagne du Siècle d'Or:

Mon honneur prime sur tout le reste ${ }^{26}$.

Le bandit : un stéréotype catalan

13 Cervantès, Lope de Vega et Tirso de Molina écrivent à une époque où paraissent de nombreux ouvrages qui décrivent la principauté catalane ainsi que le tempérament de ses habitants tout en consacrant une large part aux origines du banditisme. Il est difficile d'évaluer l'influence qu'ont pu avoir ces textes sur les écrivains du Siècle d'Or, mais l'on constate de nombreux points communs entre le bandoulier et son double littéraire, et tout particulièrement en ce qui concerne le tempérament. En 1645, le Portugais Francisco Manuel de Melo écrit que les Catalans «montrent dans les injures un fort ressentiment et c'est pourquoi ils sont plus enclins à la vengeance. Ils accordent une grande importance à l'honneur et à la parole donnée » et c'est pour cela qu'ils " abandonnent les villages pour aller vivre dans les forêts où ils hantent les chemins en se livrant sans cesse à des assauts ${ }^{27} »$. Aux dires des contemporains, la vengeance et l'honneur sont les deux principales motivations de ces personnages et les raisons d'être du banditisme. Alors qu'aujourd'hui les explications socio-économiques sont davantage privilégiées, les arbitristas du XVII ${ }^{\mathrm{e}}$ siècle cherchaient l'origine du phénomène dans des considérations environnementales. La Catalogne étant une région montagneuse, écrit Francisco de Gilabert en 1616,

[...] elle façonne nécessairement des hommes forts [...] jaloux de leur réputation et de leur honneur.

Cet enchaînement d'idées démontre l'existence d'un raisonnement logique qui explique l'origine du phénomène en s'appuyant sur des considérations structurelles. À cela s'ajoute une dimension personnelle comme Francisco Gilabert le stipule par ailleurs dans ses Discursos, «il n'y a pas de lutte de faction qui ne commence par une injure personnelle ${ }^{28} »$.

L'attachement à l'honneur, a fortiori dans une société où il est considéré comme une vertu cardinale, est un trait de caractère présent chez tous les personnages catalans qui apparaissent dans les textes, à l'exception des voleurs. Dans Antonio Roca, c'est Julia, la 
mère du héros, qui pousse son fils à la vengeance, ce qui force l'admiration du viceroi qui s'exclame :

Doña Julia,

je m'étonne de voir

qu'une si belle dame

puisse faire preuve

d'autant de fierté catalane ${ }^{29}$.

Dans le Quichotte, la parenthèse narrative de Claudia Géronima est une parfaite illustration de ce tempérament. La jeune fille apparaît peu de temps après la rencontre entre don Quichotte et Roque Guinart. Elle explique à ce dernier qu'elle est la fille d'un de ses amis Simon Forte et qu'elle vient de tuer Vincent Torrellas, le fils de Clauquel Torrellas, un ennemi de son père. Elle implore l'aide de Roque Guinart, car elle craint que « le grand nombre des parents et des amis de don Vincent n'ose tirer de lui une cruelle vengeance ${ }^{30} »$. Les motifs de Claudia mettent en évidence plusieurs aspects de son caractère. Don Vincent, explique-t-elle, l'avait courtisée et lui avait promis sa main, mais lorsqu'elle apprend quelques jours plus tard qu'il s'apprête à en épouser une autre, la sensation d'avoir été trahie éveille en elle un désir de vengeance. Impulsivement, elle part à sa recherche et

[...] sans user de plaintes ni écouter d'excuses, je lui ai déchargé cette escopette, et encore ces deux pistolets, et, à ce que je crois, lui ai mis dans le corps plus de deux balles, et fait des ouvertures par où mon honneur pût s'échapper avec son sang $g^{31}$.

Quand elle le retrouve grâce à Roque Guinart, elle lui explique qu'elle n'aurait pas agi ainsi s'il avait respecté sa parole.

Outre la vengeance, le récit de la jeune Claudia met en évidence la propension des Catalans à la colère, à l'impulsivité, ainsi que l'importance accordée à la parole donnée. Dans sa géographie de la Catalogne, Pere Gil écrit en $1600^{32}$ que les Catalans sont colériques et rancuniers, ce qui, selon lui, explique l'existence de clans rivaux, comme ceux que mentionne Claudia en se présentant à Roque Guinart.

$19 \mathrm{Au} \mathrm{XVII}{ }^{e}$ siècle, on considère en effet le banditisme comme une lutte entre différentes factions et plus particulièrement entre celles des Niarros et des Cadelles Nyerros et Cadells en catalan, deux clans dont la rivalité remonte à la fin du siècle précédent. Comme l'explique Xavier Torres, l'antagonisme initial a progressivement disparu et les rivalités se sont transmises d'une génération à l'autre par " dérivation indépendante ${ }^{33}$ ", occultant l'existence des autres groupes. Dans un mémoire adressé à Philippe III en 1615, l'arbitrista Martín Ferreira écrit ceci :

Nous informons Votre Majesté et ses ministres que toute la Principauté de Catalogne est divisée en deux clans, les Niarros et les Cadelles, et que ces deux factions entretiennent leurs anciennes inimitiés avec une telle passion héritée de leurs aïeux qu'elles s'affrontent sans raison apparente ${ }^{34}$.

Roque Guinart appartient au clan des Niarros, tout comme Claudia. Sachant cela, elle implore son aide, persuadée qu'il ne peut la lui refuser précisément à cause l'amitié qui lie le bandoulier à son père. Ces facettes du stéréotype sont reprises dans $E l$ bandolero où Pedro Armengol dit qu'il a "hérité d'un clan » parce que, comme le précise le narrateur :

Aucune nation n'est plus conservatrice en amitié et n'éprouve plus de difficulté à apaiser ses discordes : c'est là qu'est née la vengeance et c'est de cette nation qu'a été bannie la réconciliation ${ }^{35}$. 
Dans Antonio Roca, l'appartenance à un clan n'est pas mentionnée, mais plusieurs indices textuels montrent qu'il est important pour lui de se constituer un réseau. À peine fait capitaine, il dit à ses hommes :

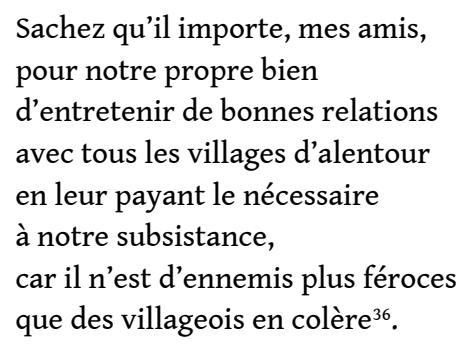

Seul, le bandit survit difficilement et il a toujours besoin de personnages adjuvants qui interviennent pour le sustenter ou pour l'aider à se soustraire à la justice. Antonio Roca en est un parfait exemple, puisqu'il ne parvient à s'enfuir de Barcelone que grâce à l'aide des habitants. Après avoir tué le baron, il se retrouve pris au piège dans une tour où il avait trouvé refuge en prenant en otage un enfant afin de menacer le vice-roi. La nuit tombée, le père de l'enfant, ainsi que quelques-uns de ses amis, aident le héros à s'échapper et à rejoindre le port pour qu'il puisse s'embarquer sur une galère à destination de Naples. Une fois à bord, il comprend que le capitaine du navire entend le livrer à la justice et grâce au soutien du sous-lieutenant, il saute par-dessus bord, rejoint le port à la nage et trouve refuge chez une habitante qui accepte de l'héberger jusqu'à l'aube sur la foi de sa seule renommée. En d'autres termes, la connivence entre le bandoulier et les populations locales apparaît comme la condition sine qua none à sa survie.

Le bandoulier semble donc bien être un stéréotype à lui tout seul, qui active dans le récit d'autres idées reçues sur la Catalogne et ses habitants, telles que la vengeance, l'amitié et l'honneur.

Adrien Roig voit dans l'idéalisation du personnage du bandoulier une manifestation de ce qu'il appelle la «catalanophilie» des auteurs castillans ${ }^{37}$. L'engouement suscité par ce personnage et par le tempérament catalan s'inscrit en effet dans un intérêt plus large des auteurs pour la Catalogne qu'ils considèrent comme le conservatoire de l'honneur espagnol. Tirso de Molina écrit ainsi dans El Bandolero que

Dans les autres provinces, la considération de l'honneur, fils adoptif du courage, est étrangère; seule la Catalogne le glorifie par nature. Là, il naît et ne meurt jamais ; du berceau jusqu'à la tombe, il accompagne fidèlement ses habitants ${ }^{38}$.

L'idée se confirme également dans les différents éloges que Cervantès a faits de Barcelone qu'il tenait pour «la fleur des belles cités du monde [...], l'honneur de l'Espagne [...], l'école de la noblesse, le modèle de la loyauté ${ }^{39}$ ». Antonio Rey Hazas voit dans les dithyrambes de Cervantès une critique $a$ contrario de la Cour madrilène et de l'autorité toute puissante qui en émane. La capitale catalane est en effet perçue par ses contemporains comme la Cité rebelle qui ose s'élever contre l'hégémonie du gouvernement ${ }^{40}$, car, comme l'écrit Francisco Manuel de Melo, les Catalans «plus que les autres nations de l'Espagne, aiment leur liberté ${ }^{41} »$.

L'interprétation du stéréotype

27 Marcel Grandière suggère que le stéréotype a pour fonction d'organiser intellectuellement la société qui les utilise à différents desseins ${ }^{42}$ : expliquer 
l'inexplicable, concevoir des modèles ou des anti-modèles. Le bandoulier, tel qu'il apparait dans les trois œuvres étudiées ici, est-il un exemple ou un contre-exemple ?

D'aucuns, à l'instar de Martín de Riquer ou encore Núria del Lucas Val ${ }^{43}$, ont évoqué la sympathie des auteurs du Siècle d'Or pour ce personnage emblématique de la Catalogne du XVII ${ }^{e}$ siècle, car ils auraient vu en eux des modèles de vertu et de justice. Cette interprétation repose sur le poncif du bandoulier au grand cœur qui n'est mû que par un désir de venger son honneur ou celui d'un proche. Mais le bandit n'en reste pas moins un criminel. Dès lors, comment expliquer qu'un tel personnage ait été élevé au rang d'exemple?

La coexistence des voleurs et du bandoulier oblige à distinguer ce dernier dans un univers criminel qui n'est pas présenté favorablement dans la narration. Les auteurs n'ont en effet pas de mots assez durs pour condamner la vie de bandit que les personnages sont généralement contraints de mener. Cervantès, dans La Galatea, écrit par exemple qu'il n'y a rien de plus «étranger à toute la chrétienté et digne d'être déploré $^{44}$ ». De surcroît, les bandouliers eux-mêmes évoquent invariablement leur insatisfaction, comme Roque Guinart qui admet « qu'il n'y a genre de vie plus inquiet ni plus agitét ${ }^{45}$ ». Et le narrateur de renchérir en précisant plus loin que c'est une «vie assurément misérable et ennuyeuse ${ }^{46}$ \%. Même Don Quichotte reconnaît que le banditisme, qu'il assimile à une maladie, n'a rien d'enviable et c'est pourquoi il tente de raisonner les hommes de Roque Guinart pour « leur persuader de laisser cette manière de vie périlleuse tant pour l'âme que pour le $\operatorname{corps}^{47} »$. Il donne un conseil similaire à Roque après que celui-ci a confessé «son espérance d'en sortir pour entrer dans un port assuré ${ }^{48} »$ :

Votre grâce est malade, elle connaît son mal et le Ciel [...] qui est votre médecin lui appliquera les remèdes qui la guériront [...] et cela d'autant plus que les pécheurs éclairés sont plus près de se corriger que les simples. [...] Si votre grâce veut abréger le chemin et se mettre aisément en celui de son salut, venez avec moi, et je vous apprendrai le métier de chevalier errant ${ }^{49}$.

Le recours aux lexiques de la médecine et de la guérison met en évidence que pour l'Ingénieux Hidalgo, le banditisme est une déviation condamnable de l'âme qui encourage des hommes aussi vertueux que Roque Guinart à mettre leurs qualités au service d'une cause indigne d'eux.

Sous ces mots se dessine une incompatibilité entre l'état de bandoulier et le tempérament de Roque Guinart. En proposant à celui-ci d'entrer dans la chevalerie errante, don Quichotte cherche à l'extraire d'une condition qui ne lui correspond pas, mais Roque Guinart répond à cette offre par une " rire », comme pour signifier qu'il n'y a aucun remède à son mal. En effet, le bandit, qui est un exclu, ne peut pas réintégrer la société et ne trouve donc son salut que dans la mort. Sur ce point, Roque Guinart semble être une exception en littérature, car il ne meurt pas ; mais le Quichotte n'est pas strictement une histoire de bandit et Roque Guinart n'est qu'un personnage secondaire que l'Ingénieux Hidalgo croise dans ses aventures catalanes. Aussi est-ce pour cela que le bandoulier s'en retourne à ses activités après avoir escorté les deux héros jusque sur la plage de Barcelone ${ }^{50}$. Dans les deux autres œuvres du corpus en revanche, le bandit ne trouve sa délivrance que dans la mort qui suit un aveu de repentance.

Dans Antonio Roca, l'entrée en scène du frère Feliciano dans la troisième journée de la pièce fait émerger le doute chez le héros qui reconnaît alors que sa passion, au sens 
étymologique du terme, l'a détourné de Dieu. Pris de remords, Antonio Roca remet sa vie entre les mains de Dieu en implorant sa miséricorde :

Entre tes mains, ineffable

Seigneur, mon souffle s'épuise ${ }^{51} \ldots$

Dans El bandolero, le thème de la repentance apparaît également: Pedro Armengol est arrêté puis pendu par son propre père à qui Jaime II avait confié la tâche de châtier son fils $^{52}$. Ayant miraculeusement survécu pendant trois jours pendu au gibet, il entre chez les mercédaires et consacre le reste de sa vie à défendre les victimes du banditisme jusqu'à ce qu'il meure assassiné par un bandoulier au cours d'une prise d'otage.

Antonio Rey Hazas a fait remarquer que les bandits les plus célèbres étaient catalans, parce qu'ils avaient été inspirés aux auteurs par des modèles historiques ${ }^{53}$. Antonio Roca, Pedro Armengol et Perot Rocaguinarda ont vécu tous les trois à des époques différentes et l'histoire de leur double littéraire est considérablement éloignée de la réalité historique. Antonio Roca était un clerc du milieu du XVI ${ }^{e}$ siècle condamné au supplice ${ }^{54}$, Pedro Armengol s'est racheté une conduite en entrant dans les ordres et la vie de Perot Rocaguinarda ressemble davantage à ce que décrit la littérature populaire qu'au bandit dont parle Cervantès. Les auteurs ont complètement récrit leur histoire pour les faire correspondre à l'archétype du bandoulier justicier qui n'écoute que ce que son honneur lui dicte. Le stéréotype du bandoulier catalan se distingue donc des autres, dans la mesure où il est construit à partir d'un ensemble d'idées reçues sur le phénomène d'une part et sur la Catalogne d'autre part. La sympathie des auteurs pour ces personnages semble avoir pour fonction de donner encore plus d'intensité à la mort exemplaire du bandit.

Antonio Roca, Pedro Armengol et Roque Guinart sont les derniers grands bandits de la littérature classique. Leurs successeurs seront plus nombreux certes, mais ils correspondront davantage au poncif du voleur, du « fils de la misère " pour reprendre l'expression de Joan Reglà ${ }^{55}$. Autrement dit, nous assistons ici à la mort d'un stéréotype littéraire qui ne survivra que dans la culture populaire. La tradition orale a su conserver jusqu'au XIX ${ }^{e}$ siècle le souvenir du bandit dans des chansons, des légendes ou des contes qui ont éveillé l'intérêt des historiens et des écrivains de la Renaixença ${ }^{56}$. Ceux-ci ont vu dans ce personnage emblématique et charismatique l'âme de la nation catalane et l'ardent défenseur des intérêts de la Catalogne face à l'oppression d'une monarchie centralisatrice. Ils ont dès lors entrepris de le réhabiliter et de «venger les bandits! Faire de ces simples voleurs des partisans regroupés sous un étendard politique ! " et ainsi contribué à la naissance du nouveau stéréotype d'un bandit nationaliste avant l'heure ${ }^{57}$.

\section{BIBLIOGRAPHIE}

I.

CERVANTÈs Miguel, Don Quichotte II, Paris, Gallimard, Folio 1901, 2006. Traduction de François de ROSSET revue par Jean CASSOU. 
ID., Les deux jeunes filles, dans Nouvelles exemplaires, Paris, Le Club français du livre, 1967, p. 241-289. Traduction de Louis Viardot.

ID., La Cueva de Salamanca, dans Entremeses, Madrid, Taurus, 1981, p. 129-142.

ID., La Galatea, dans Obras Completas, Madrid, Biblioteca de Autores Españoles, 1846, vol. 1. COROMINES Joan, Diccionari etimológic i complementari de la llengua catalana, Barcelone, Curial/La Caixa, 1992.

COVARRUBIAS Sebastián, Tesoro de la lengua castellana, Madrid, Castalia, 1995.

GIL Pere, Libre primer de la historia Cathalana en lo qual se tracta de Historia o descripció natural, ço es de cosas naturals de Cathaluña, édition de Josep IGLÉSIES, Pere Gil, S.I. (1551-1622) i la seva geografia de Catalunya, Barcelone, Institut d'Estudis Catalans, 1949.

GILABERT D'ALENTORN Francisco, Discursos sobre la calidad del Principado de Cataluña, inclinación de sus habitadores y su govierno, Lérida, Luys Manescal, 1616.

MELo Francisco Manuel de, Historia de los movimientos, separación y guerra de Cataluña, Madrid, Castalia, 1996.

MOLINA Tirso de, El Bandolero, Madrid, Castalia, 1979.

ID., Los Cigarrales de Toledo, Barcelone, Jerónimo Margarit, 1635.

VEGA Lope de, Antonio Roca o la muerte más venturosa, Madrid, Real Academia Española, 1916, vol. 1, p. 660-692.

ID., El peregrino en su patria, Madrid, Castalia, 1973.

II.

AMOSSY Ruth et HERSCHEBERG PIERROT Anne, Stéréotypes et clichés, Paris, Nathan, 1997.

BALAGUER Víctor, Historia de Cataluña y de la Corona de Aragón, Barcelone, Librería San Manero, 1860-1863, vol. 4.

CARO BAROJA Julio, Realidad y fantasía en el mundo criminal, Madrid, CSIC, 1986.

GANNIER Odile, « Éditorial : Littérature à stéréotype. Réflexions sur les combinatoires narratives », dans Loxias, $\mathrm{n}^{\circ} 17$, juin 2007.

GARCíA CÁRCEL Ricardo, Historia de Cataluña. Siglos XVI-XVII, Barcelone, Ariel, 1985, 2 vol.

GRANDIÈRE Marcel, « La notion de stéréotype », Le stéréotype. Outil de régulations sociales, Rennes, Presses Universitaires de Rennes, 2003, p. 7-12.

PI I SUNYER Carles, L'aptitud econòmica de Catalunya, Barcelone, Barcino, 1929, vol. 2.

REGLÀ Joan, Joan Serrallonga, vida i mite del famós bandoler, Barcelona, Aedos, 1961.

ID., El bandolerisme català del Barroc, Barcelone, Edicions 62, 1966.

REY HAZAS Antonio, « El bandolero en la novela del siglo de Oro » dans Juan Antonio MARTíNEZ COMECHE (coord.), Le bandit et son image au Siècle d'Or, Madrid, Casa de Velázquez/Publication de la Sorbonne, 1991, p. 201-215.

ID., « La omisión de Madrid », Anales Cervantinos, XXXI, 1993, p. 9-27.

RIQUER Martín de, Cervantes en Barcelona, Barcelone, Alcantilado, 2005. 
RoIG Adrien, « Una manifestación de la catalanofilia literaria: El catalán Serrallonga, comedia de tres ingenios ", dans Antonio Vilanova (coord.), Actas del X Congreso de la Asociación Internacional de Hispanistas, Barcelona 21-26 de agosto de 1989, vol. 2, 1992, 1053-1066.

SCHUMACHER IB Mark, « La percepción en la historia y las relaciones internacionales de la época moderna », Pedralbes, 22, 2002, p. 73-109.

SOLER I TEROL Lluís Maria, Perot Rocaguinarda: història d'aquest famós bandoler, Manresa, Sant Josep, 1909.

TORRES Xavier, Els bandolers (siglos XVI-XVII), Vic, Eumo Editorial, 1991.

ID., « Cadells » dans Jesús Mestre i Camps, Diccionari d'Història de Catalunya [1992], Barcelone, Edicions 62/El Punt,1999, p. 164-166.

\section{NOTES}

1. Selon Julio Caro Baroja, le personnage du bandolero catalan est l'héritier des latronis de la Rome antique, tantôt voleur, tantôt homme au service d'une famille noble. Voir Realidad y fantasía en el mundo criminal, Madrid, CSIC, 1986.

2. Miguel de Cervantès, Don Quichotte II [1615], Paris, Gallimard, t. 2, 2006, traduction de François de Rosset revue par Jean Cassou ; Lope de Vega, Antonio Roca o la muerte más venturosa, Madrid, Real Academia Española, 1916, vol. 1 ; et Tirso de Molina, El Bandolero [1635], Madrid, Castalia, 1979. À l'exception du Quichotte, les œuvres citées n'ont fait l'objet d'aucune traduction. C'est nous qui traduirons de l'espagnol et du catalan. 3. Miguel de Cervantès, Les deux jeunes filles dans Nouvelles exemplaires, Paris, Le Club français du livre, 1967, traduction de Louis Viardot, p.241-289. La citation est à la page 256.

4. Miguel de Cervantès, Don Quichotte II..., op. cit., p. 497.

5. Martín de Riquer, Cervantes en Barcelona, Barcelone, Alcantilado, 2005, p. 64-65.

6. Odile Gannier, « Éditorial : Littérature à stéréotype. Réflexions sur les combinatoires narratives », Loxias, 17, juin 2007.

7. Lope de Vega, El Peregrino en su patria [1604], Madrid, Castalia, 1973, p. 77.

8. Tirso de Molina, Los Cigarrales de Toledo, Barcelone, Jerónimo Margarit, 1635, fol. 240r.

9. Le banditisme a été une préoccupation constante des Catalans, ainsi que de l'administration royale dans les deux premières décennies du XVIIe siècle. Plusieurs ouvrages exposent des théories sur les origines du phénomène fortement liées au « caractère » catalan. Cela est par exemple le cas du jésuite Pere Gil dans son Libre primer de la historia Cathalana écrit en 1600, du juriste barcelonais dans le Dietari où il recense tous les événements marquants qu'il a vécus ou qui lui ont été rapportés entre 1601 et 1630. On pensera également aux Discursos que compose Francisco Gilabert d'Alentorn, publiés en 1616 à Lérida, ou encore les Pactis du juriste Joan Pere Fontanella qui dénoncent, tous deux, l'inefficacité et l'inconstitutionnalité des mesures prises par le gouvernement central qui, en 1612, interdit le port d'armes à tous les Catalans, sans distinction de classe.

10. Sebastián Covarrubias, Tesoro de la lengua castellana [1611], Madrid, Castalia, 1995. 11. Miguel de Cervantès, Don Quichotte..., op. cit., p. 507. La traduction a conservé le terme espagnol synonyme de « fraile » qui désigne un moine. 
12. Ibidem., p. 506. Le nom du bandoulier est différemment orthographié selon les œuvres. Nous choisissons ici l'orthographe proposée dans la traduction du Quichotte que nous avons utilisée, à savoir Roque Guinart.

13. Lope de Vega, Antonio Roca..., op. cit., III, p. 684a.

14. Miguel de Cervantès, Don Quichotte II..., op. cit. p. 507.

15. Miguel de Cervantès, La Cueva de Salamanca, Entremeses, édition de Jean Canavaggio, Taurus, 1981, p. 132.

16. Miguel de Cervantès, Don Quichotte II..., op. cit., p. 508-509.

17. Lope de Vega, Antonio Roca..., op. cit., p. 686a.

18. Lluís Maria Soler i Terol, Perot Rocaguinarda: història d'aquest famós bandoler, Manresa, Sant Josep, 1909, p. 119-131.

19. Lope de Vega, Antonio Roca..., op. cit., p. $665 b$.

20. Tirso de Molina, El Bandolero..., op. cit., p. 79.

21. Lope de Vega, Antonio Roca..., op. cit., p. 6806.

22. Miguel de Cervantès, Don Quichotte II..., op. cit., p. 507.

23. À propos de l'étymologie du mot, voir Joan Corominas, Diccionari etimològic $i$ complementari de la llengua catalana, Barcelone, Curial/La Caixa, 1992, vol. 1.

24. Lope de Vega, Antonio Roca..., op. cit., p. $683 b$.

25. Miguel de Cervantès, Don Quichotte II..., op. cit., p. 504.

26. Lope de Vega, Antonio Roca..., op. cit., p. 6666.

27. Francisco Manuel de Melo, Historia de los movimientos, separación y guerra de Cataluña [1645], Madrid, Castalia, 1996, p. 108-109.

28. Francisco Gilabert d'Alentorn, Discursos sobre la calidad del Principado de Cataluña, inclinacion de sus habitadores y su govierno, Lérida, Luis Manescal, 1616, Discurso Primero, § 26-30, fol. $4 v$.

29. Lope de Vega, Antonio Roca..., op. cit., p. 674a.

30. Miguel de Cervantès, Don Quichotte II..., op. cit., p. 500.

31. Idem.

32. Pere Gil, Libre primer de la historia Cathalana en la qual se tracta de Historia o descripcio natural; ço es de Cathaluña, édition de Josep Iglésies, Pere Gil, S.I. (1551-1622) i la seva geografia de Catalunya [1949], Barcelone, Institut d'Estudis Catalans, 2002. Voir plus particulièrement le chapitre intitulé « Delas varias Complexions y condicions naturals dela gent y nacio Cathalana; y de quinas maneras de Gents y Nacions es estada Cathaluna habitada », p. 267-276.

33. Xavier Torres, «Cadells » dans Jesús Mestre i Camps, Diccionari d'Història de Catalunya [1992], Barcelone, Edicions 62, 1999, p. 164-166.

34. Cité par Joan REGLÀ, Joan Serrallonga, vida i mite del famós bandoler, Barcelone, Aedos, 1961, p. 51.

35. Tirso de Molina, El Bandolero..., op. cit., p. 66.

36. Lope de Vega, Antonio Roca..., op. cit., p. 681a.

37. Adrien Roig, « Una manifestación de la catalanofilia literaria: El catalán Serrallonga, comedia de tres ingenios ", dans Antonio Vilanova (coord.), Actas del X Congreso de la Asociación Internacional de Hispanistas, Barcelona 21-26 de agosto de 1989, vol. 2, 1992, 1053-1066. Nous n'avons pu inclure cette comedia dans notre corpus, car nous ne disposions d'aucun exemplaire. Cela dit, nos conclusions concordent avec celle d'Adrien Roig.

38. Tirso de Molina, El Bandolero..., op. cit., p. 66.

39. Miguel de Cervantès, Les deux jeunes filles..., op. cit., p. 270. 
40. Antonio Rey Hazas, «La omisión de Madrid », Anales Cervantinos, XXXI, 1993, p. 9-27.

41. Francisco Manuel de Melo, Historia de los movimientos..., op. cit., p. 109.

42. Marcel Grandière, «La notion de stéréotype », Le stéréotype. Outil de régulations sociales, Rennes, Presses Universitaires de Rennes, 2003, p. 7-12.

43. Martín de Riquer, Cervantes en Barcelona, Barcelone, Alcantilado, 2005, p. 63-90 et Núria del Lucas Val, « Literatura i història. Identitats colllectives i visions de l'altre al segle XVII », Manuscrits, n²4, 2006, p. 167-192.

44. Miguel de Cervantès, La Galatea, dans Obras de Cervantes, Madrid, Biblioteca de Autores Españoles, 1846, vol. 1, p. $26 b$.

45. Miguel de Cervantès, Don Quichotte II..., op. cit., p. 504.

46. Ibidem., p. 509.

47. Ibidem., p. 503. Quoi qu'insolite, cette forme intransitive du verbe " persuader » est attestée dans les dictionnaires du français contemporain et considérée comme littéraire et vieillie. Nous reproduisons ici la traduction de l'édition de référence.

48. Ibidem., p. 504.

49. Ibidem., p. 505.

50. Ibidem., p. 510.

51. Lope de Vega, Antonio Roca..., op. cit., p.692a.

52. Dans le droit catalan médiéval, le roi ne peut condamner à mort l'un de ses sujets qu'en cas de crime de lèse-majesté. Ici, c'est le seigneur local, à savoir Alberto Armengol, qui jouit de cette prérogative.

53. Antonio Rey Hazas, «El bandolero en la novela del Siglo de Oro » dans Juan Antonio Martínez Comeche (coord.), Le bandit et son image au Siècle d'Or, Madrid, Casa de Velázquez/Publications de la Sorbonne, 1991, p. 201-215.

54. Voir Julio Caro Baroja, Realidad y fantasía en el mundo criminal..., op. cit., p. 128.

55. Joan Reglà, El bandolerisme català del Barroc, Barcelone, Edicions 62, 1966.

56. Renaixença signifie renaissance (du verbe renàixer ou renèixer) et désigne un mouvement culturel et artistique de la fin du xixe siècle propre aux Pays Catalans (Catalogne, Valence, les Îles Baléares) et aux régions occitanes du Sud-Ouest de la France qui se caractérise par un renouveau « une renaissance » de la langue catalane ou occitane. On prendra soin de ne pas confondre avec le Renaixement qui désigne la Renaissance du Xvie siècle.

57. Víctor Balaguer, Historia de Cataluña y de la Corona de Aragón, Barcelone, Librería San Manero, 1860-1863, vol. 4, p. 240.

\section{RÉSUMÉS}

Le bandit catalan est un personnage que l'on rencontre fréquemment dans la littérature espagnole du Siècle d'Or et des auteurs tels que Cervantès, Lope de Vega et Tirso de Molina se sont beaucoup intéressés à lui à une époque où le banditisme resurgit avec force en Espagne. Le poncif littéraire présente de nombreux points communs avec le stéréotype social tel qu'il est représenté dans les documents contemporains qui exposent les origines du phénomène. Il est 
dans les deux cas dépeint comme un justicier qui n'écoute que ce que lui dicte son honneur. Toutefois, il s'agit d'une vision déformée de la réalité qui pose un problème d'interprétation: comment expliquer que les auteurs espagnols du XVII siècle aient pu voir dans ce criminel un modèle de vertu?

INDEX

Mots-clés : stéréotype, bandit, Catalogne, littérature du Siècle d'Or

\section{AUTEUR}

MATHIAS LEDROIT

ATER d'espagnol à l'Université de Paris IV-Sorbonne 